\title{
A Clinical Study of Medulloblastoma in Correlation with Molecular Biology and Histological Variations in Childhood Medulloblastoma in Northeast India
}

\author{
Mrinal Bhuyan ${ }^{1}$ Hriday Haloi ${ }^{1, \odot}$ Basanta Kumar Baishya ${ }^{1} \quad$ Inamul Haque ${ }^{1}$ \\ ${ }^{1}$ Department of Neurosurgery, Gauhati Medical College and \\ Hospital, Guwahati, Assam, India \\ Address for correspondence Hriday Haloi, MS, General Surgery, House \\ No. 2, Karnalata Path, Bhetapara, Beltola, Guwahati, Kamrup (Metro) \\ 781028, Assam, India (e-mail: drhridayhaloi1985@gmail.com).
}

Indian J Neurosurg 2019;8:155-160

\begin{abstract}
Introduction Medulloblastoma is a common embryonal childhood tumor predominantly seen in children (1-3). Medulloblastoma can be classified in different histological and molecular subgroups, each of them characterized by discrete clinical presentation, demographic features, prognosis, and outcome. This may help in patient stratification, treatment strategy, design, and implementation of the targeted therapy.

Aims and Objectives We aimed to study the patients included in the study with regard to variables such as age, sex, clinical features, and various modes of management in our hospital. We also studied histological and molecular variations in the group.

Materials and Methods A prospective study was carried out in the Department of Neurosurgery, Gauhati Medical College and Hospital, Guwahati, from January 2016 to August 2017. Sixty-five patients diagnosed as medulloblastoma were included in the study. We studied the spectrum of clinical findings, radiological and pathologic characteristics, modes of surgical management, and postoperative complications. Study of molecular biology was done in the Department of Bioengineering, Gauhati University. Results and Observation Results and observations were obtained based on the age and radiological, pathological, histological, and molecular variants.

Conclusion Medulloblastoma is seen more in pediatric age group and males. Classical variant was the most common type with mostly vermian location followed by desmoplastic variant which was paravermian to lateral in location. Postoperatively,

Keywords

- medulloblastoma

- histopathology

- genetics radiotherapy and chemotherapy have proved very useful. Statistical analysis suggested a significant correlation between age groups and complications and also between histological types and associated complications. P53 mutation was noted in a section of the study population but majority cases did not show mutations in selected genes.
\end{abstract}

\section{Introduction}

Medulloblastoma is a common, malignant, embryonal childhood tumor located in the cerebellum that is predominantly seen in children but may also occur in adults. ${ }^{1-3}$ Medulloblastoma is an example of a disease that has benefitted from advances in diagnostic imaging, surgical techniques,

\section{received}

May 6, 2019

accepted

July 15, 2019

published online

October 18, 2019 radiation therapy, and combination chemotherapy over the past decades. It is composed of densely packed cells with round to oval or carrot-shaped nuclei with abundant chromatin and scanty cytoplasm..$^{4-6}$ Medulloblastoma accounts for up to $38 \%$ of all pediatric posterior fossa tumors and represents the most common pediatric posterior fossa tumor overall. ${ }^{7.8} \mathrm{By}$ far, the cerebellum is the most common location
DOI https://doi.org/

$10.1055 / \mathrm{s}-0039-1698293$

ISSN 2277-954X.
(C)2019 Neurological Surgeons'

Society of India
License terms

() (1) $\ominus \circledast$ 
for medulloblastomas (94.4\% of cases), and most ( $>75 \%$ ) of these arise in the midline cerebellar vermis., ${ }^{4,5}$ Other less common locations include the fourth ventricle (3\%), other areas of the brain $(2.1 \%)$, and the spinal cord $(0.6 \%)$. The World Health Organization classifies medulloblastoma as a grade IV lesion and recognizes five major subtypes of the tumor: classic, desmoplastic, extensively nodular with advanced neuronal differentiation, anaplastic, and large cell. Other less common subtypes include medullomyoblastoma and melanotic medulloblastoma. Using genome-based, highthroughput analytic techniques, medulloblastoma can be classified as four molecular subtypes including WNT pathway subtype, SHH pathway subtype, and two less well-defined subtypes, group 3 and group 4. Each of them is characterized by discrete clinical presentation, demographic features, prognosis, expression profiling, and genomic abnormalities. The identification of molecular subgroups has a great impact on clinical management, including patient stratification, treatment strategy, and design and implementation of targeted therapy. This study is such an attempt to classify a small subset of patients based on their histological subtypes and correlate their outcome with the molecular subtype.

\section{Aims and Objectives}

The aims and objectives are as follows:

1. To study the patients included in the study with regard to various variables such as age, sex, and other clinical features.

2. To study the various modes of management in our hospital.

3. To study histological variations in the study group.

4. To study the molecular variations in the group.

\section{Materials and Methods}

This was a prospective study performed in the Department of Neurosurgery, Gauhati Medical College and Hospital, Guwahati, from January 2016 to August 2017. A total of 65 patients diagnosed with medulloblastoma were included in the study. On admission, detailed history, clinical examinations, and all necessary investigations were done and patients were managed accordingly. We studied the spectrum of clinical findings, radiological and pathologic characteristics, modes of surgical management, and postoperative complications. A study of the molecular biology was done in collaboration with the Department of Bioengineering, Gauhati University.

Histological Examination: The different histopathological variants of medulloblastoma were differentiated using standard histological preparations (hematoxylin and eosin), which were used to assess general architectural and cytological features, including nodule formation.

Molecular Study: DNA sequencing study was done to identify mutations in the following genes: P53, IDH1, BRAF, and $\mathrm{H} 3 \mathrm{~F} 3 \mathrm{~A}$.

Statistical Analysis: Statistical analysis of data was done using Pearson's chi-square test and student's $t$-test.

Results and Observations: A total number of 65 patients were included in the study-Forty (61.5\%) were male and 25 (38.4\%) were female. The most common age group was 5 to 15 years, followed by 3 to 5 years. Forty-two (64.6\%) patients were in the age group of 5 to 15 years. As shown in - Table 1, classical was the most common histological variant in all age groups with a total of 51 (78.4\%) biopsy specimens, followed by desmoplastic with a total of $12(18.4 \%)$ samples. - Fig. 1 shows MRI of medulloblastoma. Desmoplastic variant was not found in the age group of 0 to 3 years. It was found in the study that the twp2 anaplastic variants were females.

On plain computed tomography (CT) scan of head it was found that in the classical variant, 47 (92\%) showed hyperdensity. In the desmoplastic variant, $9(75 \%)$ showed mixed density while $3(25 \%)$ showed isodensity. The two (100\%) anaplastic variants showed mixed density. In contrast, CT scan of head revealed that $43(84.3 \%)$ of the classical variant showed homogenous enhancement and 8 (15.6\%) showed heterogenous enhancement. All patients with desmoplastic and anaplastic variants showed heterogenous enhancement.

With regard to location of tumor, it was found that 49 (96\%) of classical variant was vermian in location, as shown in - Table 2. Desmoplastic tumor was found to be either paravermian in 7 (58\%) or lateral in 5 (41.6\%). Anaplastic tumor was found to be paravermian in location.

On magnetic resonance imaging (MRI), it was found that 49 (96\%) classical variants showed T2 hyperintensity, as shown in - Fig. 2. However, in the desmoplastic variant, only $2(16.6 \%)$ were found to be $\mathrm{T} 2$ hyperintense. The remaining 10 (83\%) desmoplastic variants were T2 isointense. All anaplastic variants were found to be T2 hyperintense. Classical and desmoplastic variants were found to have well-defined margins, while all anaplastic variants had poorly defined margins. Hydrocephalus was found in 45 (88\%) classical variants, 7 (58\%) desmoplastic variants, and $2(100 \%)$ patients of anaplastic variants. Hemorrhage and necrosis were found in 4 (33.33\%) desmoplastic variants and $2(100 \%)$ anaplastic variants. It was not found in any patient of the classical variant.

In our study of a total of 65 cases, 54 (83.07\%) had hydrocephalous and $51(78.4 \%)$ required medium pressure ventriculoperitoneal shunt (MPVP) shunt. Gross total excision was

Table 1 Age group and histological variant

\begin{tabular}{|l|l|l|l|l|}
\hline Age group (years) & Classical & Desmoplastic & Anaplastic & Total \\
\hline $0-3$ & 8 & 0 & 1 & 9 \\
\hline $3-5$ & 11 & 3 & 0 & 14 \\
\hline $5-15$ & 32 & 9 & 1 & 42 \\
\hline
\end{tabular}




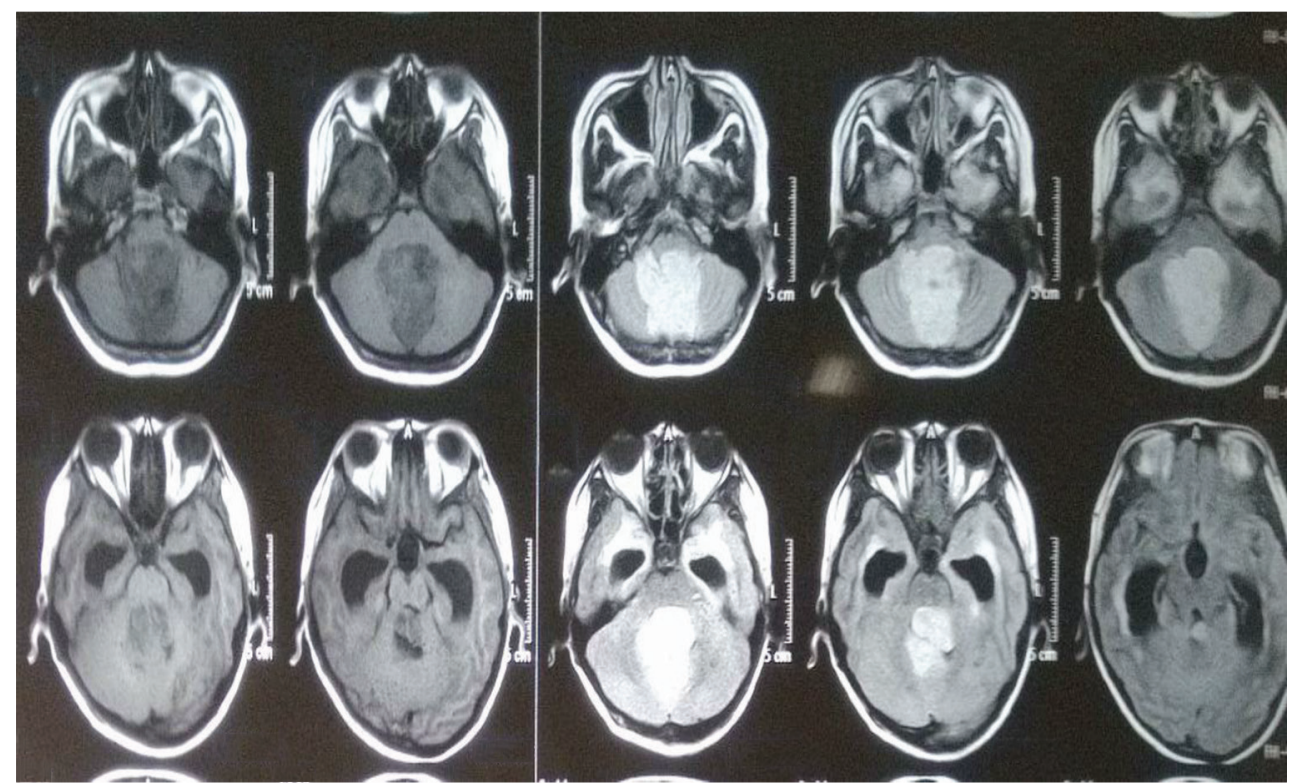

Fig. 1 T1, contrast, and T2 of the medulloblastoma case.

Table 2 Histological variant and location of tumor

\begin{tabular}{|l|l|l|l|l|}
\hline Histological variant & Vermian & Paravermian & Lateral & Total \\
\hline Classical & 49 & 2 & 0 & 51 \\
\hline Desmoplastic & 0 & 7 & 5 & 12 \\
\hline Anaplastic & 0 & 2 & 0 & 2 \\
\hline
\end{tabular}

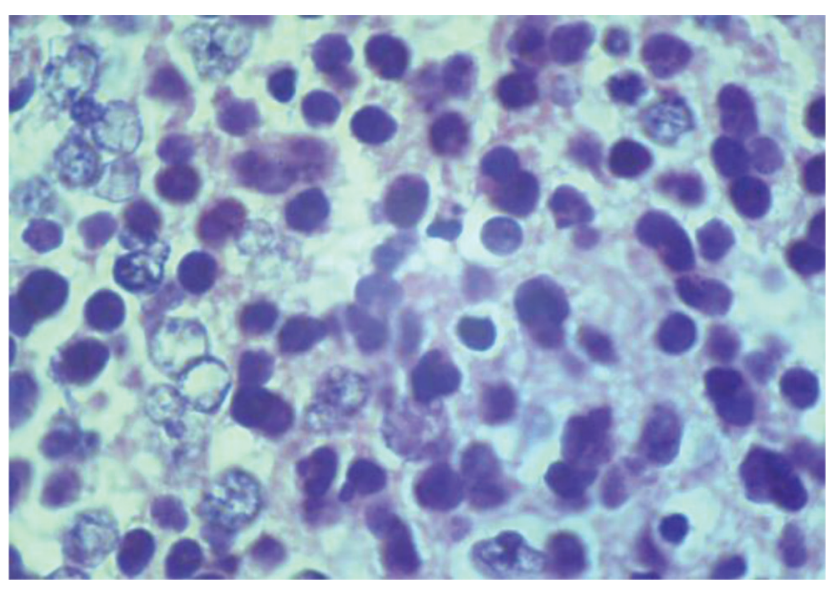

Fig. 2 Classical type of medulloblastoma.

possible in 41 (80\%) classical variants and 10 (83\%) desmoplastic variants. Anaplastic variants underwent near total excision.

One patient who presented with poor Glasgow coma scale (GCS) preoperatively expired 36 hours after surgery. Cerebellar ataxia was seen in $10(15.3 \%)$ cases and nystagmus in 10 (15.3\%) cases. Cerebellar mutism was seen in $4(6.15 \%)$ cases. Cranial nerve palsy (abducens) was seen in 3(4.6\%) cases. Recurrence was noted at 1 -year follow-up in 6 cases, of which two cases did not complete their radiotherapy cycles. In the 0 to 3 years age group, total number of patients with complications was 7 (77.7\%), in the 3 to 5 year group it was 7 (50\%), and only 3 (7\%) in the 5 to 15 years age group. After analyzing the age distribution in relation to complications of the cases using the $t$-test, the $p$-value was calculated to 0.000071 which is less than 0.05 and thus a significant correlation was seen between the age groups and associated complications. In the classical variant, complication was found in 14 (27\%); whereas 1 complication (8\%) was present in desmoplastic variant, and $2(100 \%)$ in anaplastic variants. As shown in - Table 3, after analyzing the histological variations in relation to complications of the cases using the Pearson's chisquare test the value was calculated to be 7.665 with degree of freedom 2 and $p$-value calculated to be 0.022 , which is less than 0.05 , indicating a significant association between histological types and associated complications. Also, $p$-value was calculated to be 0.020 after applying the student's $t$-test, which is less than 0.05 , indicating a significant correlation between the histological types and associated complications.

As shown in - Table 4, DNA sequencing for detection of mutation was done for four genes-P53, IDH1, BRAF, and H3F3A. Of total 65 cases in our study, mutation was noted in $P 53$ gene in 7 cases, while rest of the 58 cases did not show mutation in any of the four genes tested. It is shown in $\mathbf{- F i g s .} \mathbf{3}$ and $\mathbf{4}$.

\section{Follow-Up}

Follow-up was done at 1 month, 3 months, 6 months, 9 months, and 1 year. Recurrence was seen in 6 cases, of which 4 were of classical type and 2 anaplastic type. All six cases had near total excision and two of them did not complete their radiotherapy cycles. 
Table 3 Statistical analysis of complications in relation to histological types

\begin{tabular}{|l|l|l|l|l|l|l|}
\hline \multicolumn{7}{|c|}{ Case processing summary } \\
\hline & \multicolumn{9}{|c|}{ Cases } \\
\cline { 2 - 7 } & Valid & Missing & \multicolumn{2}{l|}{ Total } \\
\cline { 2 - 7 } & N & Percentage & N & Percentage & N & Percentage \\
\hline $\begin{array}{l}\text { Histological_TYPE_GRP* } \\
\text { Complications_GRP }\end{array}$ & 65 & $100 \%$ & 0 & $0.0 \%$ & 65 & $100 \%$ \\
\hline
\end{tabular}

\begin{tabular}{|c|c|c|c|c|c|}
\hline \multicolumn{6}{|c|}{ Histological_TYPE_GRP*complications_GRP cross-tabulation } \\
\hline & & & \multicolumn{2}{|c|}{ Complications_GRP } & \multirow[t]{2}{*}{ Total } \\
\hline & & & Negative & Positive & \\
\hline \multirow{9}{*}{$\begin{array}{l}\text { Histological__ } \\
\text { TYPE_GRP }\end{array}$} & \multirow[t]{3}{*}{ ANA } & Count & 0 & 2 & 2 \\
\hline & & \% within Histological_TYPE_GRP & $0.0 \%$ & $100.0 \%$ & $100.0 \%$ \\
\hline & & $\begin{array}{l}\text { \% within } \\
\text { Complications_GRP }\end{array}$ & $0.0 \%$ & $11.8 \%$ & $31.1 \%$ \\
\hline & \multirow[t]{3}{*}{$\mathrm{CLA}$} & Count & 37 & 14 & 51 \\
\hline & & \% within Histological_TYPE_GRP & $72.5 \%$ & $27.5 \%$ & $100.0 \%$ \\
\hline & & $\begin{array}{l}\% \text { within } \\
\text { Complications_GRP }\end{array}$ & $77.1 \%$ & $82.4 \%$ & $78.5 \%$ \\
\hline & \multirow[t]{3}{*}{ DES } & Count & 11 & 1 & 12 \\
\hline & & \% within Histological_TYPE_GRP & $91.7 \%$ & $8.3 \%$ & $100.0 \%$ \\
\hline & & $\begin{array}{l}\% \text { within } \\
\text { Complications_GRP }\end{array}$ & $22.9 \%$ & $5.9 \%$ & $18.5 \%$ \\
\hline & Count & 48 & 17 & 65 \\
\hline & & \% within Histological_TYPE_GRP & $73.8 \%$ & $26.2 \%$ & $100.0 \%$ \\
\hline & & $\begin{array}{l}\text { \% within } \\
\text { Complications_GRP }\end{array}$ & $100.0 \%$ & $100.0 \%$ & $100.0 \%$ \\
\hline
\end{tabular}

\begin{tabular}{|l|l|l|l|}
\hline \multicolumn{2}{|c|}{ Chi-square tests } & Asymp. Sig. (two-sided) \\
\hline Pearson chi-square & Value & df & 0.022 \\
\hline Likelihood ratio & 7.665 & 2 & 0.019 \\
\hline Linear-by-linear association & 7.877 & 2 & 0.021 \\
\hline No. of valid cases & 5.347 & 1 & \\
\hline
\end{tabular}

a cells (50.0\%) have expected count less than 5 . The minimum expected count is 0.52 .

\begin{tabular}{|c|c|c|c|c|c|}
\hline \multicolumn{6}{|c|}{ Symmetric measures } \\
\hline & & Value & Asymp. standard error ${ }^{\mathrm{a}}$ & Approx. $\mathrm{T}^{\mathrm{b}}$ & Approx sig. \\
\hline Interval by interval & Pearson's R & -0.289 & 0.100 & -2.397 & $0.020(c)$ \\
\hline Ordinal by ordinal & Spearman correlation & -0.278 & 0.099 & -2.298 & $0.025(c)$ \\
\hline \multicolumn{2}{|l|}{ No. of valid cases } & 65 & & & \\
\hline
\end{tabular}

aNot assuming the null hypothesis.

bUsing the asymptotic standard error assuming the null hypothesis.

'Based on normal approximation.

\section{Discussion}

In our study, the incidence in different age groups and male to female ratio of 1.6:1 is similar to findings reported by Agerlin et al, with a male to female ratio of $2: 1 .^{9}$ The incidence of different clinical features obtained in our study is similar to that reported in the study by Alston et al..$^{10}$ In our study, classical variant (78.4\%) was the most common, followed by desmoplastic (18.4\%) and anaplastic (3.2\%) variants. This is slightly different to the findings obtained in the study by McManamy et al's ${ }^{11}$ (classical variant in $71 \%$ cases, desmoplastic in $16 \%$ cases, and anaplastic in $17 \%$ cases). 
Classical variants appeared as homogenous mass in CT scan while the desmoplastic and anaplastic variants appeared as heterogenous mass. We found that the classical variants were mostly vermian in location, desmoplastic was both paravermian and lateral, and anaplastic was paravermian. Two anaplastic variants were paravermian in location. Bourgouin et $\mathrm{al}^{12}$ have reported that vermian medulloblastoma mostly appear as a hyperdense mass on plain CT scan, and with intense homogenous enhancements with contrast. Classical variant showed T1 hypointensity and T2 hyperintensity, while the desmoplastic group in T1 showed variable hypo- to isointensity and mostly isointensity in T2 MRI signal. Similar findings were reported by Bourgouin et al. ${ }^{12}$

Table 4 Distribution of gene mutation

\begin{tabular}{|l|l|}
\hline Mutated gene & No. of cases \\
\hline P53 & 7 \\
\hline IDH1 & 0 \\
\hline BRAF & 0 \\
\hline H3F3A & 0 \\
\hline
\end{tabular}

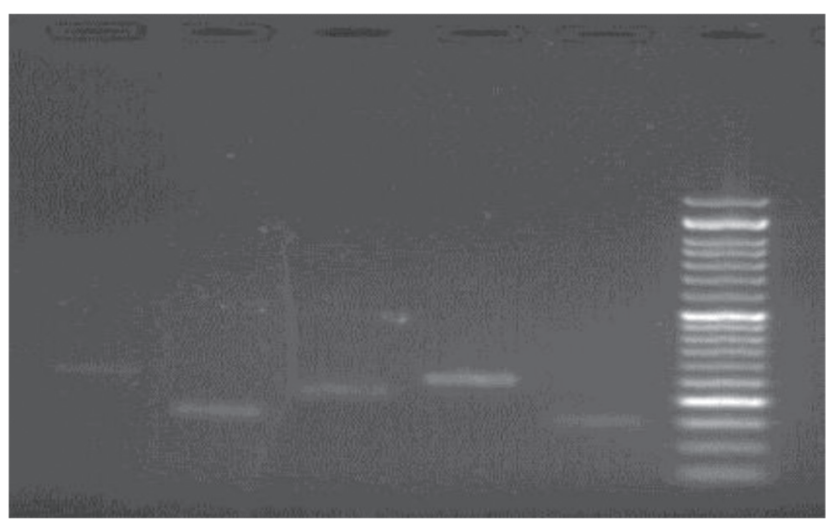

Fig. 3 Gel electrophoresis of p53 (exons 5-9).
All cases of anaplastic variant had edema, followed by 49 (96.07\%) cases of classical and 10 (83.33\%) cases of desmoplastic having edema. Forty-seven $(92.1 \%)$ cases of classical variant had well-defined margins, followed by 10 (83.33\%) cases of desmoplastic variant. All cases of anaplastic variants had poorly defined margins.

In our study, of the total 65 cases, 54 (83.07\%) had hydrocephalous and 51 (78.4\%) required ventriculoperitoneal shunt. Gross total excision was done in 51 (78.46\%) cases and near total was done in 14 (21.5\%) cases. Out of the 14 near total excision cases, 8 had extension to the brain stem.

Postoperatively, cerebellar ataxia was seen in 10 (15.3\%) cases and nystagmus in 10 (15.3\%) cases. Cerebellar mutism was seen in $4(6.15 \%)$ cases. Cranial nerve palsy (abducens) was seen in 3 (4.6\%) cases. Recurrence was noted in six cases, of which two cases did not complete their radiotherapy cycles.

In our study, the 0 to 3 years age group had 7 (10.7\%) cases with complications, the 3 to 5 years age group had 7 (10.7\%) cases with complications, and the 5 to 15 years age group had $3(4.6 \%)$ cases with complications. Applying the student's $t$-test, $\mathrm{p}$-value was calculated to be 0.000071 , which is less than 0.05 and thus a significant correlation was seen between the age groups and associated complications.

In our study, the classical variant group had 14 (21.5\%) cases with complications, desmoplastic group had 1 (1.5\%) cases with complications and anaplastic group had 2 (3.07\%) cases with complications. Applying the students $t$-test, $\mathrm{p}$-value was calculated to be 0.020 , which is less than 0.05 , indicating a significant correlation between the histological types and associated complications.

\section{Conclusion}

Through our study, we reach to the following conclusions:

- Medulloblastoma is commonly seen in pediatric age group and more common in males.

- Within the pediatric age group, certain variants appeared in the younger group while others in the older group.

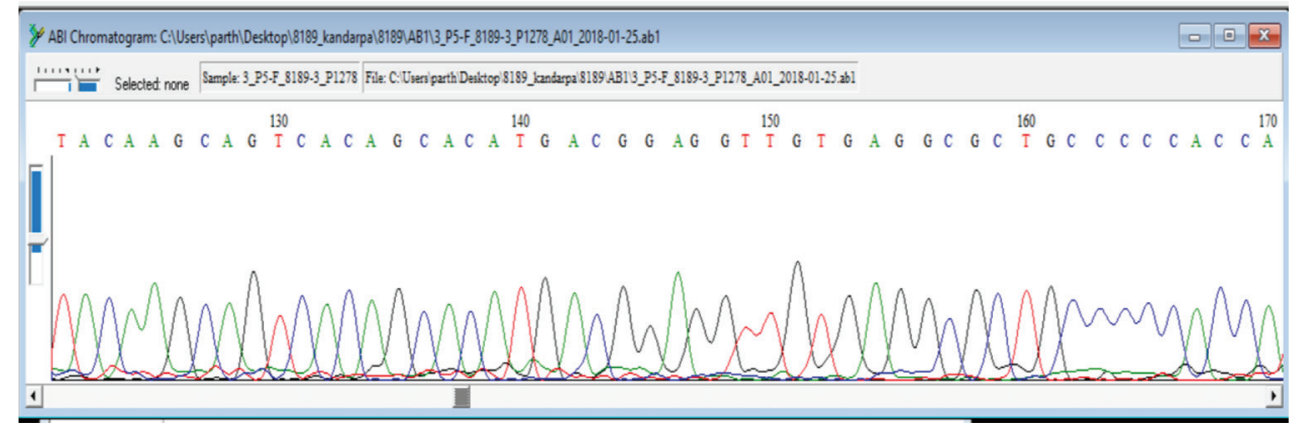

Fig. 4 Electropherogram of exons 5 to 6 of the p53 gene sequence. 
- Patients usually presented with features of raised intracranial pressure (ICP), imbalance, and visual disturbance.

- Classical variant was the most common type with mostly vermian location, followed by desmoplastic variant which was paravermian to lateral in location.

- CT and MRI features varied in different variants of medulloblastoma.

- In most cases, gross total excision was possible and surgical outcome was favorable.

- Postoperatively, radiotherapy and chemotherapy proved successful.

- Recurrence was seen to be associated with the level of surgical excision and postoperative radiotherapy and chemotherapy.

- Statistical analysis suggested a significant correlation between age groups and complications and also between histological types and associated complications.

- P53 mutation was noted in a section of the study population but majority of cases did not show mutations in the selected genes.

- A wider pool of gene study is required for complete molecular profiling of the study population.

\section{Conflict of Interest}

None declared.

\section{References}

1 Roberts RO, Lynch CF, Jones MP, Hart MN. Medulloblastoma: a population-based study of 532 cases. J Neuropathol. Exp Neurol 1991;50(2):134-144
2 Giangaspero F, Bigner SH, Kleihues P, Pietsch T, Trojanowski JQ. Medulloblastoma. In: Kleihues P, Cavenee W, eds. Pathology and Genetics of Tumours of the Central Nervous System. Lyon, France: International Agency for Research on Cancer; 2000: 129

3 Duffner PK, Cohen ME. Recent developments in pediatric neuro-oncology. Cancer 1986;58(2, Suppl):561-568

4 Bailey P, Cushing H. Medulloblastoma cerebelli: a common type of midcerebellar glioma of childhood. Arch Neurol Psychiatry 1925;14:192-224

5 Rubinstein LJ, Northfield DW. The medulloblastoma and the so-called "arachnoidal cerebellar sarcoma." Brain 1964;87:379-412

6 Kleihues P, Cavenee W, eds. Pathology and Genetics of Tumors of the Nervous System. Lyon, France: International Agency for Research on Cancer Press; 2000

7 Arseni C, Ciurea AV. Statistical survey of 276 cases of medulloblastoma (1935-1978) Acta Neurochir (Wien) 1981;57(3-4):159-162

8 Farwell JR, Dohrmann GJ, Flannery JT. Central nervous system tumors in children. Cancer 1977;40(6):3123-3132

9 Agerlin N, Gjerris F, Brincker H, et al. Childhood medulloblastoma in Denmark 1960-1984. A population-based retrospective study. Childs Nerv Syst 1999;15(1):29-36,discussion36-37

10 Alston RD, Newton R, Kelsey A, Newbould MJ, et al. Developmental Medicine and Child Neurology. London, UK: 2003;45(5):308

11 McManamy CS, Pears J, Weston CL, et al; Clinical Brain Tumour Group. Nodule formation and desmoplasia in medulloblastomas-defining the nodular/desmoplastic variant and its biological behavior. Brain Pathol 2007;17(2):151-164

12 Bourgouin PM, Tampieri D, Grahovac SZ, Léger C, Del Carpio R, Melançon D. CT and MR imaging findings in adults with cerebellar medulloblastoma: comparison with findings in children. Am J Roentgenol 1992;159(3):609-612 